\title{
Peptide Synthesis in Ionic Liquids (PEPSIL): All You Need is in the Toolbox!
}

Khouloud Jebri ${ }^{\mathrm{a}, \mathrm{b}}$, Nicolas Galy ${ }^{\mathrm{a}}$, Viacheslav Zgonnik ${ }^{\mathrm{a}}$, Marie-Rose Mazières ${ }^{\mathrm{a}}$, Yves Génisson ${ }^{\mathrm{a}}$, Frédéric Guillen ${ }^{\mathrm{a}}$, Taïcir Ben Ayed ${ }^{\mathrm{b}}$, Michel Baltas ${ }^{\mathrm{a}}$, Jean-Christophe Plaquevent ${ }^{\mathrm{a} *}$

${ }^{a}$ Université Paul Sabatier, CNRS-UMR 5068, SPCMIB, 118 route de Narbonne, $\underline{F}$-31062 Toulouse Cedex 9, France

${ }^{\mathrm{b}}$ Institut National des Sciences Appliquées et de Technologie (INSAT), Université de Carthage, Tunis, Tunisie plaquevent@chimie.ups-tlse.fr

Keywords: ionic liquids, peptide synthesis, amino acids, coupling agent, cyclopeptides.

This personal account describes our studies regarding peptide synthesis in ionic liquids (PEPSIL). In addition, we summarize our experiments in which the use of ionic liquids gives simple and promising access to unnatural amino acids in an enantiomerically enriched form. Extension of PEPSIL method to complex structures such as cyclopeptides is also discussed. Some of these cyclopeptides are the direct precursors of bioactive and pharmaceutical compounds.

\section{Introduction}

The strong potential of ionic liquids for organic synthesis has been demonstrated since the beginning of the century $[1,2]$. As soon as appeared the seminal contributions in this field, we imagined that the use of ionic solvents would be especially promising for amino acids and peptides chemistry, which remains a major challenge for bio-organic and pharmaceutical applications [3, 4]. This account highlights our studies regarding peptide synthesis in ionic liquids (PEPSIL). In addition, experiments in which the use of ionic liquids gives simple and promising access to unnatural amino acids in an enantiomerically enriched form are summarized. Extension of PEPSIL method to complex structures such as cyclopeptides is also discussed: some of these cyclopeptides are the direct precursors of bioactive and pharmaceutical compounds. In the final part, our discovery of a new coupling agent allowing the development of waste-free peptide coupling is disclosed.

\section{Results and discussion}

\section{Synthesis and resolution of unnatural amino acids}

The access to unnatural amino acids via Strecker reactions in ionic media has been described by several groups [5-8]. According to the authors, the expected amino nitriles were obtained in high yields by means of three component reactions between aldehydes, amines, and trimethylsilyl cyanide. The ionic 
liquids were recycled and reused several times without loss of reactivity.

Since these syntheses obviously yielded the racemic mixtures of the compounds, we immediately wondered if ionic solvents could be helpful for their enantiomeric resolution. In this respect, we rapidly focused on enantioselective liquid-liquid extraction (ELLE) as a promising and innovative method for this purpose.

ELLE method combines in a single technique the concepts of resolution (i.e.: enantiomeric separation of a racemic mixture) and of biphasic extraction [9]. The success of such a procedure implies two important requisites:

i. The generation of diastereomeric complexes of the extractant with the enantiomers, formed by means of intermolecular interactions (ion pairing, hydrogen bonding, $\pi-$ $\pi$ interactions, dipolar and Van der Waals interactions).

ii. The existence of two (at least partially) immiscible phases.

This type of process presents high scalability, recyclability and easy handling. ELLE nowadays can be combined with multistage countercurrent cascade technology. The main challenge in the field is the development of chiral hosts, which must be easy to obtain and able to display high selectivity for a wide range of substrates. In this context, we assumed that chiral ionic liquids (CILs) could be excellent candidates since they could act both as the solvent and the chiral selector. Also, using a hydrophobic CIL layer, combined with water extraction, would perfectly match the principles of green chemistry.

The first example of CILs being simultaneously used as the solvent and the chiral selector in ELLE process was reported by Tang et al [10] with enantiomeric excess up to $50 \%$ for a single-step extraction. Enantioselective enrichment of racemic amino acids was achieved through ligand-exchange via copper complexes of CILs.

Simultaneously, we examined a possible alternative process of ELLE without using any metal ion. In this context we were wondering about the outcome of the chiral anion of the CIL, like a tartrate moiety provided by our methodology [11], when exposed to a racemic counter-ion. Could we expect chiral recognition?

We first examine pipecoloxylidide as model substrate [12]. The $(S)$ enantiomer of this molecule is the precursor of a family of local anaesthetics. The first step was the preparation of the corresponding racemic $\mathrm{NTf}_{2}$ salt, obtained in quantitative yield (Figure 1).

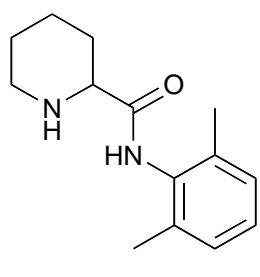

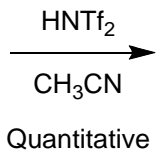

$(R S)$-pipecoloxylidide

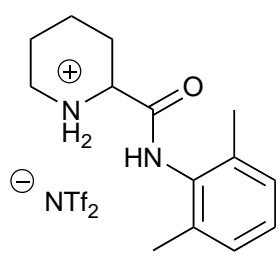

(RS)-[HPip][NTf $]$ 
Figure 1. Preparation of the starting racemic pipecoloxylidide salt.

The biphasic chiral extraction system was created by mixing the chiral ionic liquid $\left[\mathrm{PBu}_{4}\right]_{2}[(R, R)-\mathrm{Trtr}](\mathrm{Trtr}=$ tartrate, Figure 2$)$ prepared according to [11], and the racemic substrate $(R, S)$-[HPip][ $\left.\mathrm{NTf}_{2}\right]$ in the commercial hydrophobic IL [omim][ $\left.\mathrm{NTf}_{2}\right]$. The IL phase was mixed for 2 hours in order to allow the chiral host and the racemate to interact. Water was then added and the biphasic system was stirred for an additional 12 hours. Thanks to the gap in hydrophilicity between the various ions present in the mixture, we observed a complete ion metathesis: the hydrophobic tetrabutylphosphonium and bis-triflimide ions combined in the IL phase, while the hydrophilic tartrate and ammonium ions preferred the aqueous layer. The overall process resulted in the selective extraction of the $(S)$-enantiomer of pipecoloxylidide, which is precisely the eutomeric stereoisomer in this series (Figure 2).

Worthy of note is that incubation time and temperature control at $50^{\circ} \mathrm{C}$ were required, as ee drops if incubation is performed at lower or higher temperature, and no ee is observed without incubation. Thus, the best conditions yielded $30 \%$ ee for a single extraction.

This is the first example of the use of chiral ionic liquids in ELLE method in the absence of any metallic ions. Full results for this study were reported elsewhere [13].
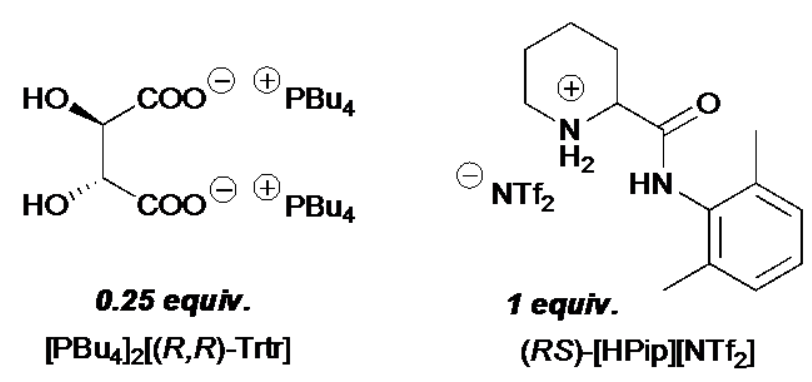

1. [omim] $\left[\mathrm{NTf}_{2}\right]$

2. water extraction

Water Layer

(S)-[HPip][(R,R)-Trt]

$30 \%$ ee

IL Layer

$2\left[\mathrm{PBu}_{4}\right]\left[\mathrm{NTf}_{2}\right]$

(R)-[HPip][NTf $]$

\section{Peptide synthesis in ionic liquids (PEPSIL)}

The different aspects of peptide synthesis in molecular solvents have been extensively reviewed [14-16]. Nevertheless, no reports of the controlled homogeneous chemical peptide coupling in ionic liquids were disclosed in the literature before 2004. Yet, we expected this approach to be of great potential because of the solubility of amino acid derivatives in ionic media. We also believed that the coupling could be favored by using hydrophobic ionic liquids, as well as by stabilization of the charged coupling reagents and reaction intermediates in these media [17-20].

We thus examined peptide coupling in ionic liquids with the modern coupling agents HATU and BOP that we selected for their 
structural similarities with ionic solvents (Figure 3). We expected those reagents to be easily dissolved and stabilized in ionic liquids, as well as their inherent by-products (Figure 3). As a consequence, they may provide slow but selective reactions.

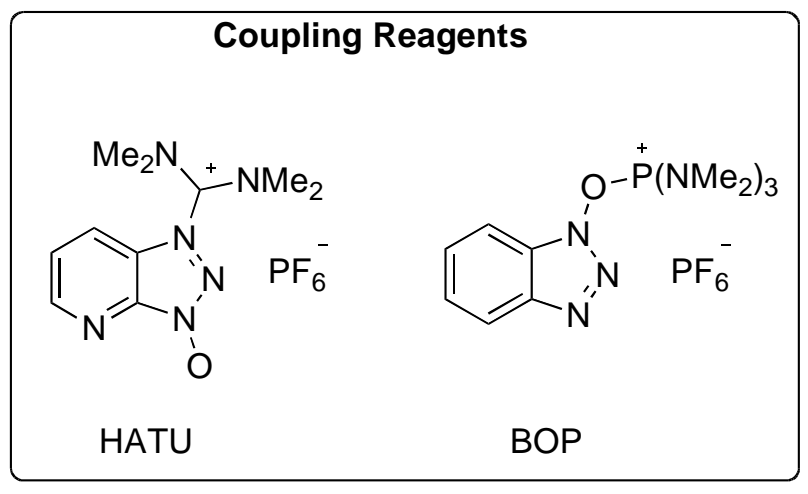

tetramethylurea

Figure 3. Coupling reagents and their residual components.

We decided to first focus on dipeptide formation via coupling of quaternary $\alpha$-amino acids, which are generally more reluctant than their tertiary proteinogenic congeners (Figure 4). After optimization of the reaction conditions and extraction process, good yields were observed, which compared favorably with yields in molecular solvents such as dichloromethane or THF [17-20]. Interestingly, crude dipeptides were obtained with higher purity in ionic liquids. This confirmed our hypothesis of a more selective chemical pathway due to the stabilization effect of the ionic liquid on both the coupling reagent and the charged intermediates.

$$
\begin{aligned}
& \text { HATU or BOP }
\end{aligned}
$$

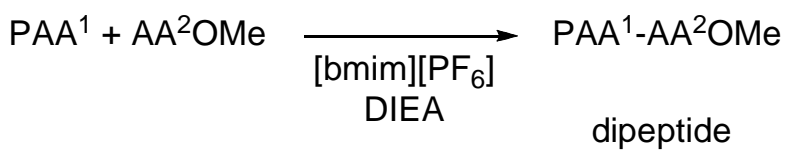

$A A^{1} \& A A^{2}:$
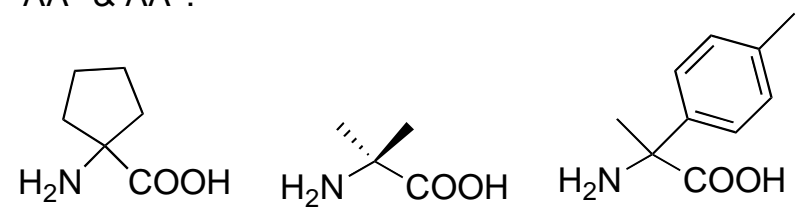

Figure 4. Dipeptide synthesis in $[\mathrm{bmim}]\left[\mathrm{PF}_{6}\right]$

After the publication of our preliminary results [17], three independent studies confirmed the interest of peptide coupling in ionic solvents, and highlighted the main conclusions of our studies. Yen and Chu described the synthesis of diketopiperazines in [bdmim] $\left[\mathrm{PF}_{6}\right]$, under microwave irradiation [21]. Sega and coworkers published the synthesis of the dipeptide Cbz-Phe-Ser-OMe in 90\% yield [22]. More recently, Poletti and Giannini extended the method to the unprecedented use of ionic liquids in the synthesis of peptide nucleic acids (PNA) oligomers, including the application of the supported ionic liquid phase (SILP) technique 
[23].

Having in hands the proof of concept, we decided to embark on the synthesis of longer and more elaborated peptidic sequences [20, 24]. Indeed, standard peptide couplings still show some drawbacks such as a rather low productivity in solid-phase methods, as well as limited chain construction in homogeneous synthesis. Thus, a promising study was to evaluate the construction of longer peptide sequences, including cyclopeptides that are generally more demanding. We first prepared the tetrapeptide $\mathrm{Z}$-(Gly- $(R)-\mathrm{MPG})_{2}-\mathrm{OMe}$ using HATU as coupling reagent and the previously prepared dipeptides, according to the same protocol we previously used for the synthesis of dipeptides (Figure 5).

ZGly-MPG + Gly-MPG-OMe

HATU, DIEA [bmim] $\left[\mathrm{PF}_{6}\right]$ $65^{\circ} \mathrm{C}$

ZGly-MPG -Gly-MPG-OMe $83 \%$

Figure 5. Synthesis of tetrapeptide Z-(Gly- $(R)-\mathrm{MPG})_{2}-$ OMe.

We were delighted to observe a high conversion, better than in THF [20, 24]. Two different samples of the protected tetrapeptide were then individually deprotected on the $N$ terminal position and on the $C$-terminal one using standard procedures, in quantitative and $85 \%$ yield respectively. Those two fragments were then coupled using HATU as activating agent. The corresponding linear octapeptide was easily secured in $83 \%$ yield (Figure 6), a similar result to the one observed in THF [20,24].
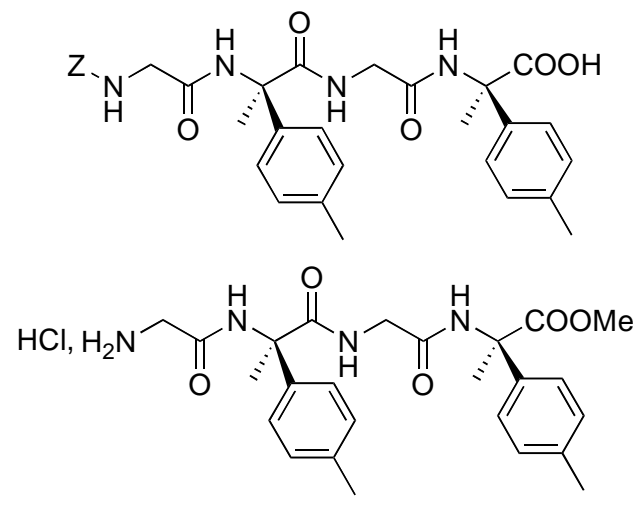

HATU/DIEA $[\mathrm{bmim}]\left[\mathrm{PF}_{6}\right]$

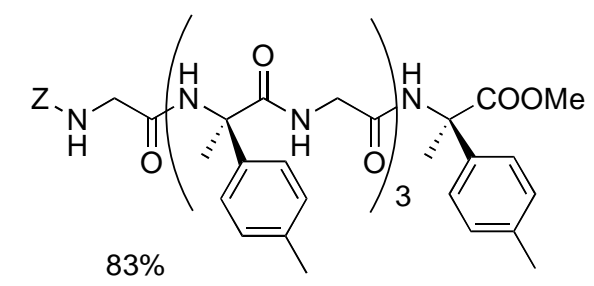

Figure 6. Synthesis of linear octapeptide Z-(Gly- $(R)$ $\mathrm{MPG})_{4}-\mathrm{OMe}$.

Each of the terminal functions of the octapeptide were then deprotected, giving the required precursor for cyclisation. Both deprotection steps proved efficient with ca $80 \%$ yields [20, 24].

The final step of this study was the cyclisation reaction yielding the corresponding cyclooctapeptide (Figure 7 ) in $[\mathrm{bmim}]\left[\mathrm{PF}_{6}\right]$ as solvent. Dimerization is a common issue in macrocyclisation reactions that is generally circumvented by means of high dilutions. However, since ionic liquids are highly viscous 
liquids, presenting a very low diffusivity, we showed that the reaction could proceed at higher concentrations of the linear octapeptide than in molecular solvents $\left(10^{-2} \mathrm{M}\right.$ in $[\mathrm{bmim}]\left[\mathrm{PF}_{6}\right]$ vs $10^{-4} \mathrm{M}$ in DMF). Indeed, using BOP as the coupling agent achieved an exciting result, delivering the targeted cyclopeptide in $85 \%$ yield [20, 24].

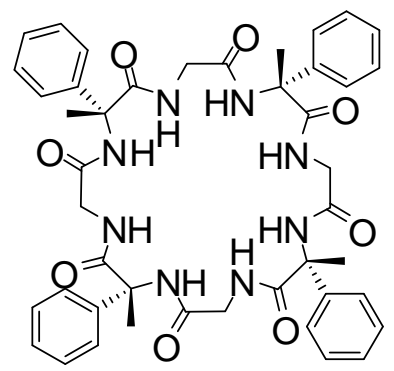

Figure 7. Targeted cyclo(Gly- $(R)-\mathrm{MPG})_{4},(\mathrm{cGM})$.

As highlighted before, one of the most interesting aspects of PEPSIL is the high purity of the crude material. This is due, at least in part, to the ability of ionic liquids to retain in solution the residual components of the coupling agents (HOAt, tetramethylurea, HMPA, Figure 3).

Our most recent application of PEPSIL method is the synthesis of cyclopeptides containing either biaryl ether or aryl-aryl linkages [25]. These compounds have attracted considerable interest because of the significant biological activities that most of them exhibit, including antimicrobial and cytotoxic activities [26]. However, the synthesis of these molecules remains a genuine challenge [27]. Two representative examples of these structures are shown in Figure 8.

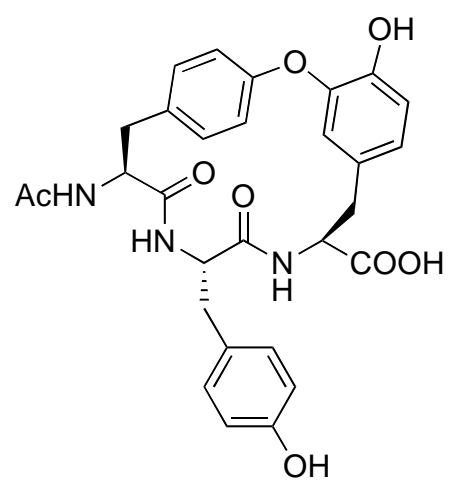

$\mathrm{K}-13$

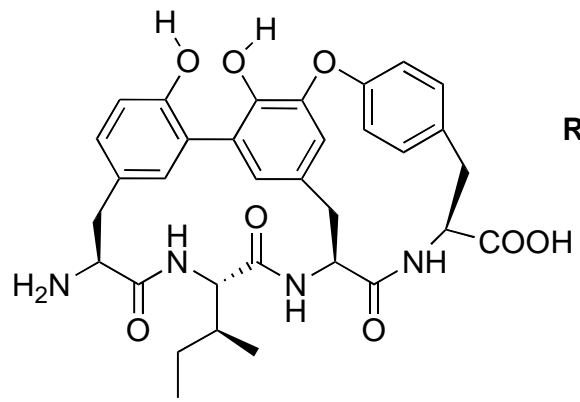

RP-66453

Figure 8. Bioactive cyclopeptides derived from tyrosine.

The biaryl ether moieties come from the side chains of tyrosine. Several di- and tripeptides derived from tyrosine have thus been synthesized in various ionic liquids. The most convenient one is $[\mathrm{bmim}]\left[\mathrm{PF}_{6}\right]$, due to its hydrophobicity facilitating the extraction of the synthesized peptides. Some typical results are depicted in Figure 9 [25]. 
<smiles>CC(=O)OC(Cc1ccc(O)cc1)NC(=O)C(Cc1ccc(O)cc1)N[Na]</smiles>

Yld (HATU): $37 \%$

Yld (BOP): $56 \%$<smiles>COc1ccc(CC(N)C(=O)NC(Cc2ccc(O)cc2)C(C)=O)cc1I</smiles>

Yld (HATU): $40 \%$ Yld (BOP): $89 \%$<smiles>COc1ccc(CC(NC(=O)CNC(=O)C(NC(=O)c2ccccc2)C(C)(C)C)c2ccc(OC)cc2)cc1</smiles>

YId (HATU): $76 \%$ Yld (BOP): $85 \%$

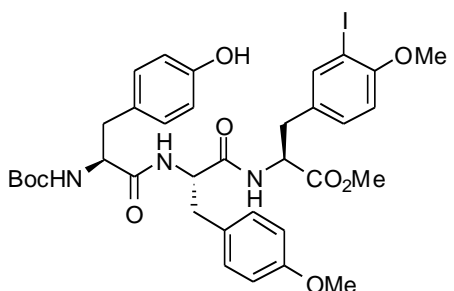

Yld (BOP): $78 \%$

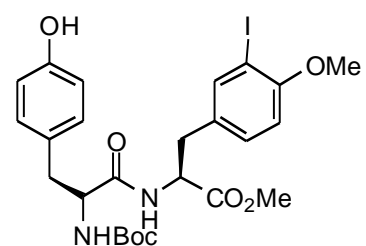

Yld (HATU): $48 \%$ Yld (BOP): $72 \%$

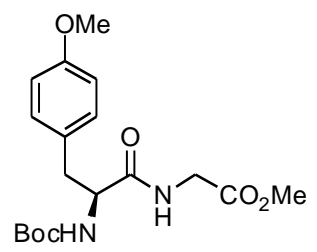

Yld (HATU): $43 \%$ Yld (BOP): $81 \%$

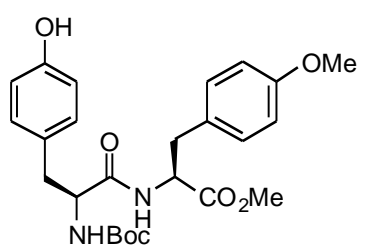

Yld (BOP): $72 \%$

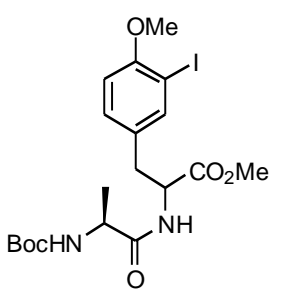

Yld (BOP): $78 \%$
Figure 9. Di- and tripeptides obtained from tyrosine in [bmim $]\left[\mathrm{PF}_{6}\right]$.

Again we observed better results when using BOP instead of HATU as coupling reagent, a behavior that we previously discussed [24]. To the best of our knowledge, those results are the first PEPSIL examples for amino acids bearing a functionalized side chain. Full details for this study will be published elsewhere [25, 28].

3. CBEIT: a new coupling reagent for waste-free PEPSIL method
The results reported so far highlighted the strong potential of PEPSIL method. Nonetheless, we thought that the use of ionic liquids in peptide synthesis could be even more advantageous than just playing the role of new solvents.

Indeed, we are now using an ionic coupling reagent with ionic liquids in a protocol where the only by-products are carbon dioxide and ethylimidazolium triflate, a proper ionic liquid that can play the role of the solvent. This innovative self-regenerating process is part of our current studies about synthesis in ionic liquid only (SILO) [29]. Such an approach, which paves the way to waste-free peptide synthesis, is of particular interest in the context of sustainable chemistry.

Our inspiration came from Rapoport group's results published some years ago [30, 31]. The authors described 1,1'-carbonylbis-(3methylimidazolium) triflate (CBMIT, Figure 10) as an efficient coupling reagent. The generated by-products were only carbon dioxide and methylimidazolium triflate ([HMIM][TfO]). As this salt is not a room temperature ionic liquid (high melting point and viscosity), we looked for a similar reagent displaying comparable reactivity while giving a by-product being liquid at low temperature. In this regard, we showed that the homologous CBEIT gave as resulting salt the ionic liquid $[\mathrm{H}-$ EIM][TfO], with a low melting point of $8^{\circ} \mathrm{C}$ and an acceptable viscosity [32, 33]. 
Figure 11. Synthesis of CBEIT.

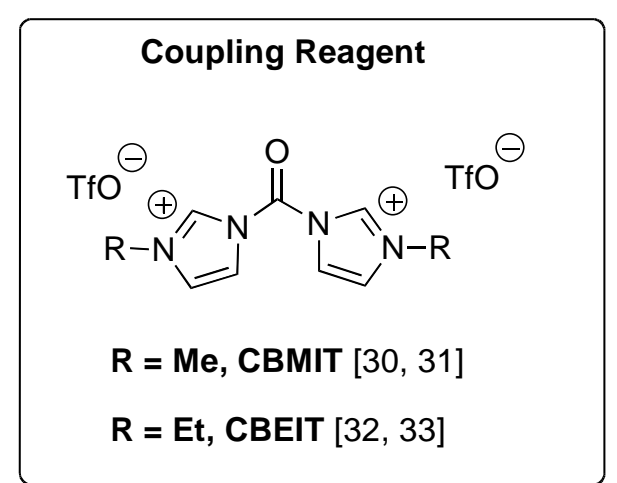

\section{Corresponding by-product}<smiles>[R]n1cc[nH+]c1</smiles>

$\mathbf{R}=\mathbf{M e},\left[\boldsymbol{H}\right.$-MIM][TfO], $\mathrm{mp}: \mathrm{ca} 100^{\circ} \mathrm{C}$ $\mathbf{R}=\mathbf{E t},\left[H\right.$-EIM][TfO], $\mathrm{mp}: 8^{\circ} \mathrm{C}$

Figure 10. Ionic coupling reagents and their corresponding by-products.

CBEIT was readily obtained by reaction of CDI with ethyl triflate, with quantitative yield and no need for further purification before use (Figure 11).

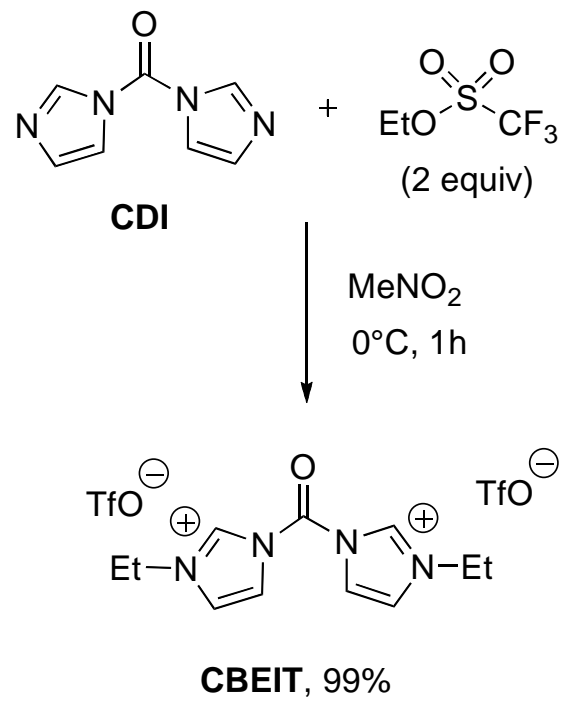

We then focused on a general method giving only carbon dioxide and $[H$-EIM] $[\mathrm{TfO}]$ as by-products. To achieve this goal, we needed to prepare the starting protected amino acids in a way avoiding any other by-products. As a result, we prepared a series of unprecedented methyl amino ester triflates by direct alkylation of amino acids with methyl triflate $(C$ protection, Figure 12). In parallel experiments, benzyl alcohol and $t$-butanol were reacted, first with CBEIT, and in turn with amino ester salts in the presence of ethyl imidazole to give the corresponding carbamates in good yields $(\mathrm{N}$ protection, Figure 12).

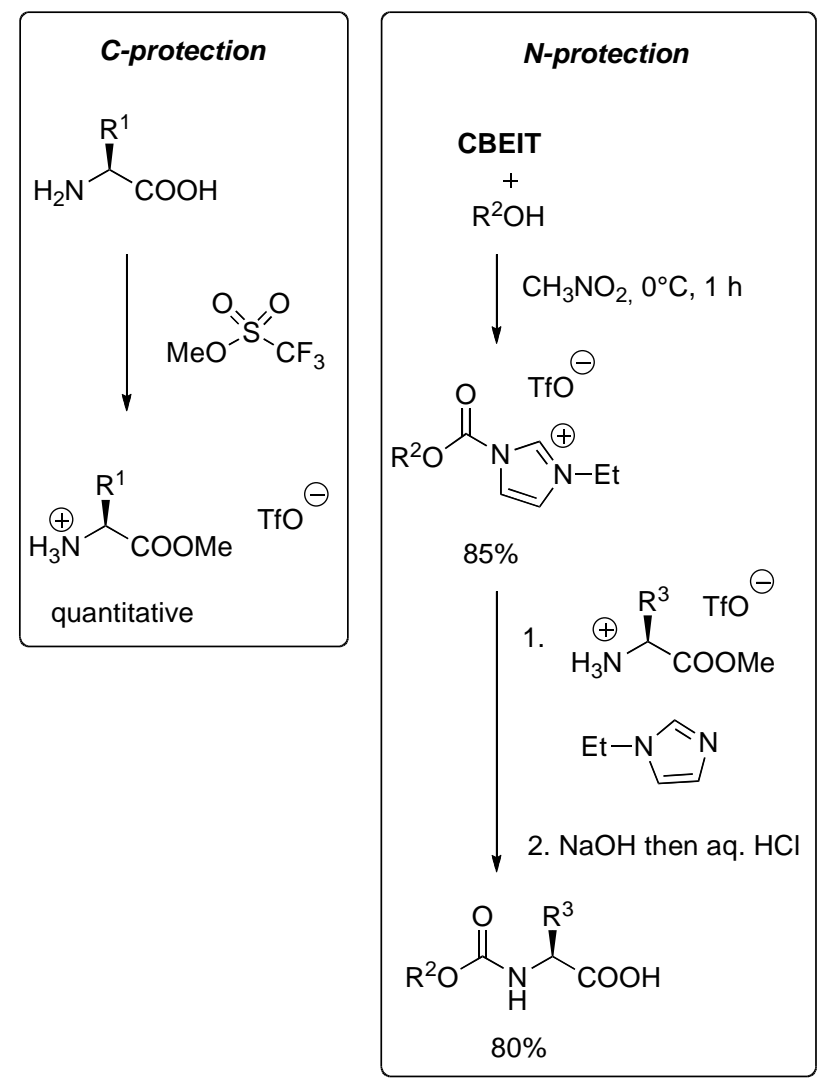

Figure 12. $N$ - and $C$-protection of starting amino acids. 
The key step was the coupling of $C$ - and $N$-protected amino acids using CBEIT as the coupling agent and ethyl imidazole as a base able to liberate the amino ester salt. Two representative results are shown in Figure 13. The dipeptides were obtained with yields as high as $75 \%[32,33]$.

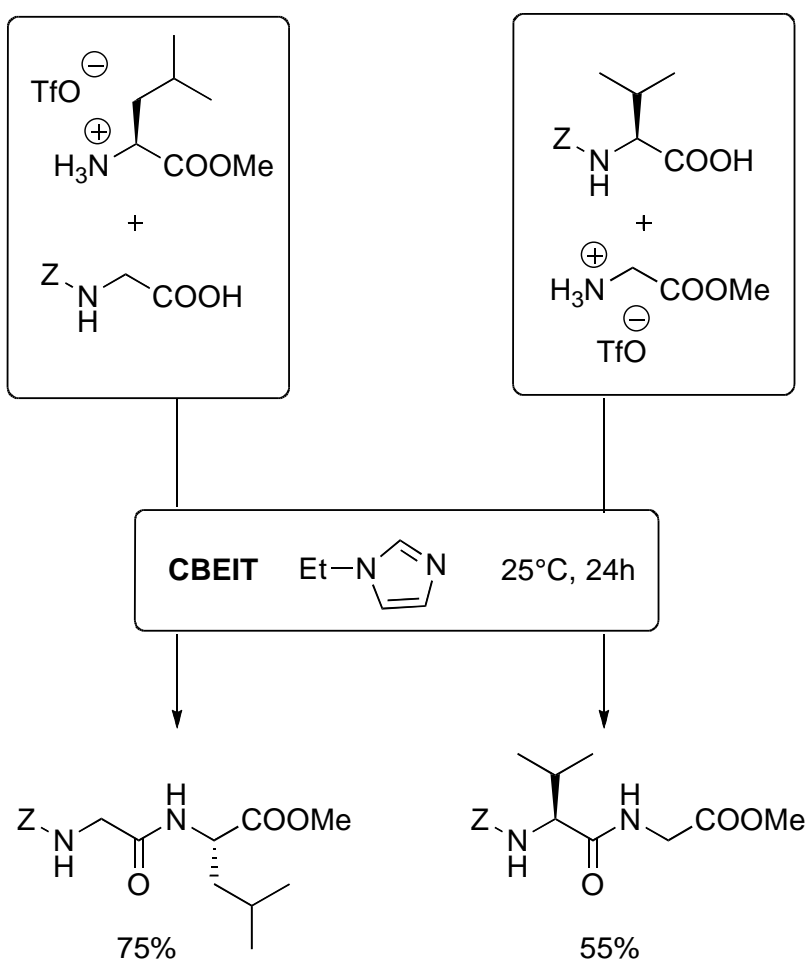

Figure 13. "Waste-free" peptide couplings.

We checked that no racemization occurred during the process $[32,33]$. The ionic liquid $[H-$ EIM][TfO] was recovered and purified by standard procedure in ca $70 \%$ un-optimized yield. Figure 14 summarizes the overall strategy for this new waste-free peptide synthesis.

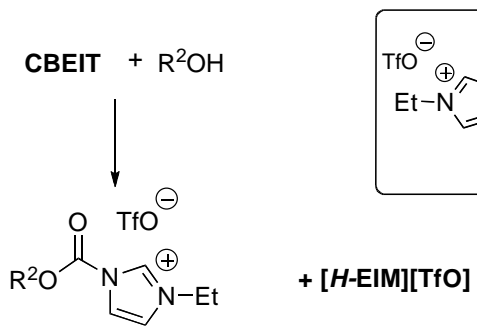

$\stackrel{\mathrm{H}}{\mathrm{N}}_{\mathrm{COOH}}^{1}$

[H-EIM][TfO], lonic Liquid $\mathrm{Et}-\mathrm{N} \aleph_{\mathrm{N}}^{\oplus} \mathrm{H}$

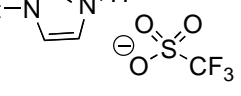

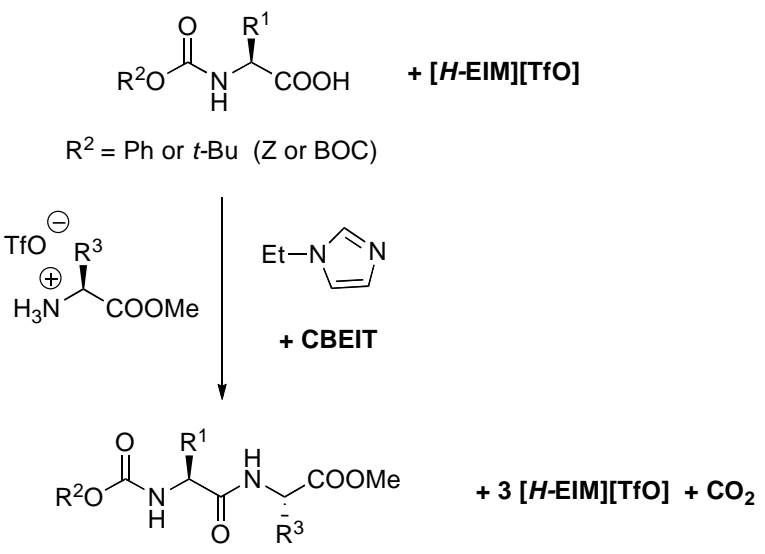

Figure 14. Overall protocol for waste-free peptide synthesis.

\section{Conclusions}

In conclusion, this account summarizes three innovative approaches for peptide chemistry using ionic liquids, while more exhaustive data in this field were reviewed elsewhere [19, 34, 35].

First, ELLE using CILs is demonstrated as a very promising and eco-friendly method for liquid-liquid resolution of amino acids.

Second, PEPSIL proves to be a powerful alternative for peptide synthesis. The construction of elaborated structures is disclosed, showing the high potential of the method.

Third, CBEIT is an excellent coupling reagent for efficient and "waste-free" peptide 
synthesis. The only by-products are carbon dioxide and an ionic liquid that may act as the solvent. Not only the peptide coupling itself is realized in such conditions, but also the necessary protections of the starting amino acids.

When considered together, these three studies highlight the rich potential of ionic liquids as tools and solvents for peptide chemistry. Although these approaches are still in infancy, we believe that future insights and discoveries in this field will afford powerful alternative methods for amino acids chemistry and peptide synthesis.

\section{Acknowledgements}

We want to express our gratitude for financial support to the following institutions:

INTENANT and the grant FP7-NMP2SL2008-214129 by the European Union,

the "PHC Utique" program of the French Ministry of Foreign Affairs and Ministry of Higher Education and Research and the Tunisian Ministry of Higher Education and Scientific Research (CMCU project number 14G1209),

Rhodia, CNRS and Paul Sabatier University.

\section{References}

[1] T. Welton, Chem. Rev. 1999, 99, 2071.

[2] J.P. Hallet, T. Welton, Chem. Rev. 2011 , 111,3508 .

[3] S.H.B. Kent, Ang. Chem. Int. Ed. 2006 , $45,4234$.
[4] S.H.B. Kent, Chem. Soc. Rev. 2009, 38, 338.

[5] J.S. Yadav, B.V.S. Reddy, B. Eshwaraiah, M. Srinivas, P. Vishnumurthy, New J. Chem. 2003, 27, 462 .

[6] M.M. Mojtahedi, M.S. Abaee, H. Abbasi, J. Iran. Chem. Soc. 2006, 3, 93.

[7] J. Akbari, C. R. Chimie 2012, 15, 471.

[8] M.K. Kathiravan, A.B. Salake, A.S. Chothe, A.N. Kale, M.M. Kulkarni, S.T. Jankar, P.J. Mundhe, P.B. Dudhe, P.L. Phalke, S.R. Kavade, Chemistry Journal 2012, 2, 199.

[9] B. Schuur, B.J.V. Verkuijl, A.J. Minnaard, J.G. de Vries, H.J. Heeres, B.L. Feringa, Org. Biomol. Chem. 2011, 9, 36.

[10] F. Tang, Q. Zhang, D. Ren, Z. Nie, Q. Liu, S. Yao, Journal of Chromatography A. 2010, 1217, 4669.

[11] A. Rouch, T. Castellan, I. Fabing, N. Saffon, J. Rodriguez, T. Constantieux, J.C. Plaquevent, Y. Génisson, RSC Advances 2013, 3, 413.

[12] The idea to examine this substrate was suggested by Dr Martin Hedberg (AstraZeneca) during the Intenant European 7th Framework Research Program For a special issue summarizing the main results of the Intenant network, please see: Org. Proc. Res. Dev. 2012, $16,277$.

[13] V. Zgonnik, C. Zedde, Y. Génisson, M.R. Mazières, J.C. Plaquevent, Chem. Commun., 2012, 48, 3185.

[14] F. Albericio, Curr. Opin. Chem. Biol. 2004, 8, 211.

[15] A. Isidro-Llobet, M. Alvarez, F. Albericio, Chem. Rev. 2009, 109, 2455.

[16] A. Ayman El-Faham, F. Albericio, Chem. Rev. 2011, 111, 6557.

[17] H. Vallette, L. Ferron, G. Coquerel, A.C. Gaumont, J.C. Plaquevent, Tetrahedron Lett. 2004, 45, 1617. 
[18] H. Vallette, L. Ferron, G. Coquerel, F. Guillen, J.C. Plaquevent, Arkivoc 2006, 200.

[19] J.C. Plaquevent, J. Levillain, F. Guillen, C. Malhiac, A.C. Gaumont, Chem. Rev. 2008, 108, 5035.

[20] J. Ternois, L. Ferron, G. Coquerel, F. Guillen, J.C. Plaquevent, ACS Symposium Series 2010, $1038,13$.

[21] Y.H. Yen, Y.H. Chu, Tetrahedron Lett. 2004, 45, 8137.

[22] L.R. Lampariello, D. Peruzzi, A. Sega, M. Taddei, M. Lett. Org. Chem. 2005, 2, 136.

[23] L. Poletti, C. Giannini, Tetrahedron 2013, 69, 1940.

[24] L. Ferron, F. Guillen, S. Coste, G. Coquerel, P. Cardinaël, J. Schwartz, J.M. Paris, J.C. Plaquevent, Tetrahedron 2011, 67, 6036.

[25] K. Jebri, dissertation 2016, in preparation.

[26] L. Feliu, M. Planas, Int. J. Peptide Res. Therapeutics 2005, 11, 53.

[27] T. Gulder, P.S. Baran, Nat. Prod. Rep. 2012, 29, 899.

[28] K. Jebri, T. Ben Ayed, M. Baltas, F. Guillen, unpublished results.

[29] K. Jebri, M.R. Mazières, S. Ballereau, T. Ben Ayed, F. Guillen, M. Baltas, J.C. Plaquevent, Tetrahedron Lett. 2014, 55, 1353.

[30] A.K. Saha, P. Schultz, H. Rapoport, J. Am. Chem. Soc. 1989, 111, 4856.

[31] F.S. Gibson, H. Rapoport, J. Org. Chem. 1995, 60, 2615.

[32] N. Galy, M.R. Mazières, J.C. Plaquevent, Tetrahedron Lett. 2013, 54, 2703.

[33] N. Galy, J.C. Plaquevent, L'Actualité Chimique 2015, №392, XX.

[34] A.A. Tietze, P. Heimer, A. Stark, D. Imhof, Molecules 2012, 17, 4158.

[35] A.A. Tietze, F. Bordusa, R. Giernoth, D. Imhof, T. Lenzer, A. Mass, C. Mrestani-Klaus, I.

\section{3, 14, 4044.}

